\title{
Triggering processes of microseismic events associated with water injection in Okuaizu Geothermal Field, Japan
}

\author{
Kyosuke Okamoto ${ }^{1 *}$ (D, Li Yi $^{1}$, Hiroshi Asanuma', Takashi Okabe ${ }^{2}$, Yasuyuki Abe ${ }^{3}$ and Masatoshi Tsuzuki ${ }^{4}$
}

\begin{abstract}
A continuous water injection test was conducted to halt the reduction in steam production in the Okuaizu Geothermal Field, Japan. Understanding the factors triggering microseismicity associated with water injection is essential to ensuring effective steam production. We identified possible triggering processes by applying methods based on microseismic monitoring, including a new method to determine the presence of water in local fractures using scattered $P$-waves. We found that the evolving microseismicity near the injection point could be explained by a diffusion process and/or water migration. We also found that local microseismicity on a remote fault was likely activated by stress fluctuations resulting from changes in the injection rate. A mediator of this fluctuation might be water remaining in the fracture zone. After the injection was terminated, microseismicity possibly associated with the phase transition of the liquid was found. We conclude that a variety of triggering processes associated with water injection may exist.
\end{abstract}

Keywords: Geothermal reservoir, Microseismic monitoring, Triggering process, Seismic scattering

\section{Introduction}

During water injection into geothermal reservoirs, it is important to monitor and control migration of the water to avoid cooling of the reservoir and to recover steam efficiently. For this purpose, microseismic monitoring has been widely used to estimate the spatiotemporal behavior of reservoirs (e.g., Fehler et al. 1987; Baria et al. 1999). Asanuma et al. (2008) determined the locations of microseismic events in a seismic cluster in Basel, Switzerland, with a 20-m error using the double-difference (DD) method (Waldhauser and Ellsworth 2000) and suggested that the precise locations (errors on the order of $10^{1} \mathrm{~m}$ ) of pore-pressure migration can be estimated from the evolution of microseismic events. The assumption of diffusion in fluid-saturated rocks (Shapiro et al. 1997) established a relationship between microseismic events and the water front (Shapiro et al. 2002). In the diffusion

\footnotetext{
*Correspondence: okamoto.kyosuke@aist.go.jp

${ }^{1}$ Renewable Energy Research Center, Fukushima Renewable Energy Institute, National Institute of Advanced Industrial Science and Technology, 2-2-9 Machiikedai, Koriyama, Fukushima 963-0298, Japan Full list of author information is available at the end of the article
}

model, microseismic events are thought to be triggered by linear pore-pressure relaxation due to a Biot slow wave propagating through a poroelastic fluid-saturated medium. Shapiro and Dinske (2009) developed a theory regarding a linear/nonlinear diffusion process. In addition, several studies attempted to clarify pore-pressure migration by including the selective water flow in permeable fractures and faults (hereafter referred to as the water flow model) (e.g., Evans et al. 2005; McClure and Horne 2011; Mukuhira et al. 2016).

To evaluate possible triggering processes, determination of the presence or absence of water and its migration around microseismic events is necessary. However, this cannot be determined directly without real-time excavation, which is seldom feasible owing to cost and time limitations. Knowledge of the presence or absence of water is also important in assessing the effect of water injection on the original seismicity (e.g., whether and how injection affects its activity). Such estimation enables us to distinguish fluid-triggering events and background natural seismicity and should lead to a better understanding of the relationship between them. We employed 
high-resolution mapping of microseismic events as well as a new method to determine the presence or absence of water in fractured rocks using the scattering of direct $P$-waves. Combining these results with information on the well-bottom pressure and temperature, we were able to discern the behavior of the injected water and estimate possible triggering processes for the microseismicity associated with injection.

\section{Outline of the injection test and microseismic monitoring in the Okuaizu Geothermal Field}

As part of the "Technology to Evaluate and Manage Geothermal Reservoirs" project conducted by the Japan Oil, Gas and Metals National Corporation (JOGMEC) (e.g., Okabe et al. 2016), a continuous water injection (without pressurization; the average wellhead pressure during injection was $0.17 \mathrm{MPa}$ ) test was conducted in the Okuaizu Geothermal Field, Fukushima, Japan, from June to August 2015 in an effort to stop the reduction in steam production. The total amount of injected fluid was approximately $1.2 \times 10^{5} \mathrm{~m}^{3}$. During the initial stage of the test (days $0-60$ ), the injection rate was $50 \mathrm{~m}^{3} / \mathrm{h}$ $\left(1.39 \times 10^{-2} \mathrm{~m}^{3} / \mathrm{s}\right)$; in the final stage (day 60 to the end of injection), the rate was $70 \mathrm{~m}^{3} / \mathrm{h}\left(1.94 \times 10^{-2} \mathrm{~m}^{3} / \mathrm{s}\right)(\mathrm{see}$ Fig. 1a). It took roughly 10 days from the start of injection before the injection rate was stabilized to $50 \mathrm{~m}^{3} / \mathrm{h}$. Figure $1 \mathrm{~b}-\mathrm{d}$ shows the injection well-called the Test Well in this study-and the injection points, which are indicated by the green line and blue circles, respectively. The injection target area was a heating zone in the geothermal field.

Five stations on the surface and four stations in boreholes (shown in Fig. 1b-d) have been deployed around the Test Well since 2015 as part of the project. These stations possess three-component broadband accelerometers or velocimeters. Continuous seismic records are transmitted to a National Institute of Advanced Industrial Science and Technology server in real time, and hypocenters are routinely determined by manual selection of $P$-wave arrivals. The residuals in $P$-wave travel times for the estimated hypocenters are, at most, $100 \mathrm{~ms}$ $\left(\sim 10^{2}-\mathrm{m}\right.$ error in the spatial domain). The lower limit of the detectable local seismic magnitude is approximately - 2.0. During the injection test, roughly 1000 microseismic events were detected.

\section{Hypocenter determination by the double-difference method and an overview of the detected microseismic clusters}

We employed cluster analysis of microseismic events to determine the precise locations of the events associated with the injection test. The analysis was applied over three periods: (1) for 1 month before the start of injection
(Phase 1), (2) for 3 months during injection (Phase 2), and (3) for 3 months after the injection ended (Phase 3). Clusters of microseismic events were identified by cross-correlation of the waveforms (e.g., Schaff et al. 2004) of the up-down (UD) component in the $40-80 \mathrm{~Hz}$ frequency range of the borehole sensors. The relative difference in the time of arrival of the $P$-waves in a cluster was calculated on the basis of the cross-correlation to create the input for the DD method. The spatial residuals were on the order of $10^{1} \mathrm{~m}$ after the hypocenters were relocated; the centroid of a cluster was that for routine hypocenter determination.

The microseismicity that occurred in Phase 1 in an area northeast of the Test Well (see Fig. 1b) is considered to be on an existing fault [the geometry of the fault is as specified in New Energy Development Organization (1997)]. Two seismic clouds (Mqs1 and 2; see Fig. 1c) were seen in Phase 2. The microseismic events in Mqs1 occurred near the injection point in the initial stage of Phase 2 and subsequently spread toward a shallower part. Mqs2 likely occurred on the existing fault on which the microseismic events were concentrated in Phase 1. In Phase 3, the seismicity of Mqs1 and 2 became low, and Mqs3 occurred in an area northwest of the Test Well (see Fig. 1d).

\section{Analyses to estimate triggering processes of the microseismic clusters}

Our cluster analysis results suggest that the occurrence of Mqs1 and the activation of Mqs2 were probably associated with the injection. However, the triggering processes for these occurrences and for Mqs3, which was newly activated after the injection ended, are still unknown. We examined the possibility that either the diffusion model or the water flow model could explain the triggering factors for Mqs1-3. Stress fluctuations due to injection rate changes and the phase transition of water were also considered possible triggering factors in our analyses. However, other factors such as chemical reactions and thermal effects were not considered.

\section{Methods}

We calculated the spatiotemporal distribution of the events to evaluate the diffusion model $[r=\sqrt{4 \pi D t}, r$ distance, $t$ time, $D$ hydraulic diffusivity, Shapiro et al. (1997)]. Because it is difficult to suppose that the entire reservoir is isotropic, owing to the presence of faults and fractures, we applied the diffusion equation to clusters Mqs1, 2, and 3. The injection point was set as the origin $(r=0)$ for all the clusters.

The spatiotemporal variation in the presence of water needs to be examined to evaluate the water flow model. In this model, we reveal selective water flow through fractures or faults. We estimated the changes in elastic 
(a)
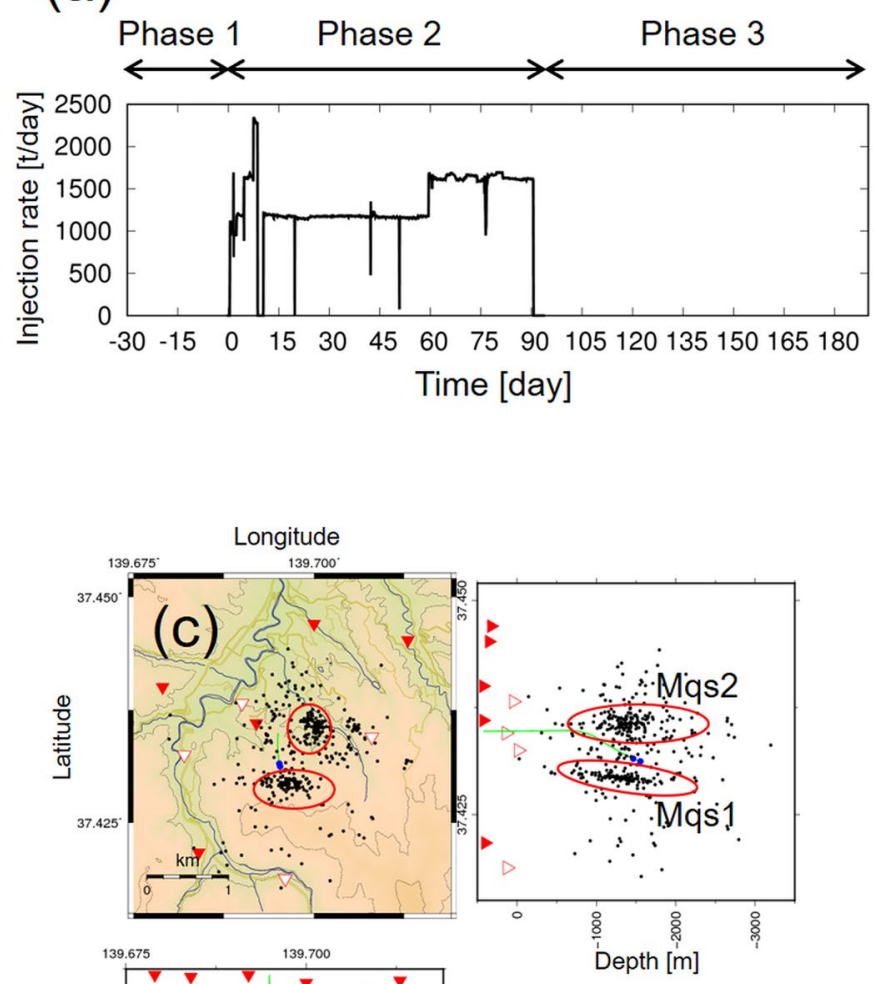
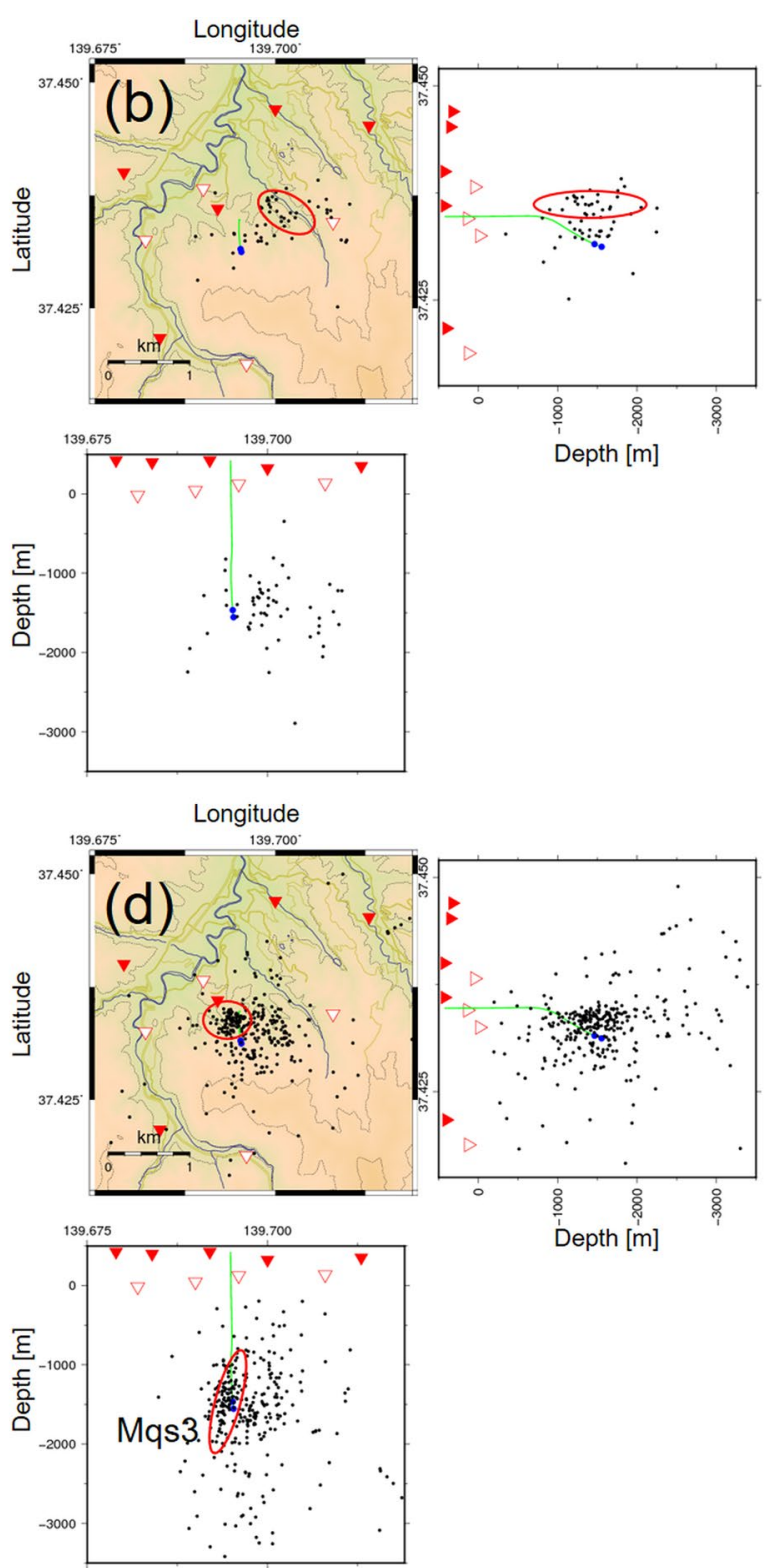

Fig. 1 Injection rate and hypocenter maps for microseismic events. a Injection rate of water. The horizontal axis shows the number of days from the start of injection; b-d hypocenter maps for $\mathbf{b}$ before the start of injection (Phase 1), c during injection (Phase 2), and $\mathbf{d}$ after the end of injection (Phase 3). The red and white triangles mark seismic stations on and below the surface, respectively. The green line shows the trace of the injected well (Test Well); the blue circles show the injection points

wave velocity in fractured rocks using the variation in seismic waveforms before and after water injection and investigated whether water was present. We employed a theoretical method to calculate the $P$-waveforms of a target earthquake on the basis of the Born approximation considering PP, PS, and SP scattered waves (Sato 1984). In the calculation, a fault and station pair can be deployed at arbitrary locations in a randomly inhomogeneous semi-infinite medium (Yoshimoto et al. 1997).
The fault parameters (strike, rake, and slip angles) and seismic magnitude are given. The inhomogeneous medium is described by the spatial autocorrelation function $R(r)=\varepsilon^{2} \exp (-r / a)$, where $r$ is the distance, $\varepsilon$ is the strength of the fluctuation of the elastic parameters, and $a$ is the correlation distance of the fluctuation. We consider that the local heterogeneous conditions are a function of the parameters $\varepsilon$ and $a$ ( $a$ is determined by $k a=1$, where $k$ is the wavelength). P codas can be synthesized 
from the theoretical power spectral densities for PP, PS, and SP conversion. By fitting theoretical waves to the observed waves, the parameter $\varepsilon$ can be estimated [details appear in Okamoto and Tsuno (2015)]. In this study, we assumed that $\varepsilon=V_{P}^{\text {inc. }} / V_{P}^{\text {back }}$, and we estimated the changes in $V_{P}^{\text {inc. }}$ associated with the injection. Here, $V_{P}^{\text {inc. }}$ and $V_{P}^{\text {back }}$ are the elastic wave velocities in fractured rocks and in the background medium, respectively. An example of the fitting results is shown in Fig. 2a, b. An earthquake that occurred during the injection period was used for this example. A 0.4-s time window starting at $0.1 \mathrm{~s}$ from the $P$-wave onset was used (component: UD, frequency band: $40-80 \mathrm{~Hz}$ ). The $0.1-\mathrm{s}$ delay was used to avoid contamination by the near-field effect because the theoretical calculation assumes the far-field term. The spatial distribution of the sensitivity of the $P$-waves was calculated by cross-correlation of the original waveform, and its time-reversed signal propagated from a target receiver, which is considered a fictitious source (e.g., Tromp et al. 2005). The sensitivity in the time window is mainly restricted to the area around the hypocenter and the station (Fig. 2c). Because the variation associated with the injection may not affect the area near the surface (e.g., near the stations), we assume that changes in $V_{P}^{\text {inc. }}$ indicate variations in the medium around the hypocenter.

We also conducted a diffraction stack migration of reflection waves in $\mathrm{S}$ codas to estimate boundaries with high-impedance contrast, such as the background medium and fractures. For the water flow model, the existence of fractures (paths for water migration) is an important factor. A constant velocity model was used in the calculation. The semblance value of a certain hyperbolic trajectory in seismic traces is applied as a weighting factor of the conventional migration to enhance the imaging results (Hollman et al. 1999). When the values along the hyperbolic trajectory differ from each other, it will return a small value. The factor can enhance only the stacking values from the real reflectors while reducing the artifacts. Considering that the errors appear as distortion of the summation curves with inaccurate positions in the simplified velocity model, it is predicted that their effect is related to the pulse duration of the extracted signal. Our simulation results show that for a 0.1- to 0.2-s pulse duration, a position error of less than $300 \mathrm{~m}$ will not affect the imaging result significantly [details are given in Yi et al. (2017)].

\section{Results using the diffusion model}

The results for Mqs1 and 2, which occurred in Phase 2, are shown in Fig. 3a. The microseismic events in Mqs1 were consistent with a diffusion process $\left(D=1.0 \times 10^{-2} \mathrm{~m}^{2} / \mathrm{s}\right)$. The evolution of the microseismic events can be explained by pore-pressure relaxation by a Biot slow wave. Saturated water appeared to exist around Mqs1. The microseismic events in Mqs2 could be explained by a diffusion process $\left(D=2.8 \times 10^{-2} \mathrm{~m}^{2} / \mathrm{s}\right)$. A characteristic tendency in Mqs2 is that they occurred intermittently, in contrast to those in Mqs1. The microseismic events in Mqs3, which appeared in Phase 3 at a different location from Mqs1 and 2, seem to be explained by a diffusion process $\left(D=1.8 \times 10^{-2} \mathrm{~m}^{2} / \mathrm{s}\right.$, Fig. $\left.3 \mathrm{~b}\right)$. All the clusters can be explained by the diffusion process. Further, Mqs2 and 3 have characteristic tendencies. The events in Mqs2 appeared to be associated with injection rate changes (Fig. 3c). The seismicity of Mqs3 became high after the injection ended. Those tendencies will be further discussed in a later section.

\section{Results using the water flow model}

Using an earthquake in the reservoir in Phase 1 as the reference, changes in $V_{P}^{\text {inc. }}$ were estimated for seven earthquakes in Phase 2 and eight earthquakes in Phase 3. Because the fault parameters are needed for this estimation, the number of available earthquakes was limited. $V_{P}^{\text {inc. }}$ increased by approximately $30 \%$ within Mqs 1 and $10 \%$ within Mqs2 relative to the reference (Fig. 4a). It seems that gas in the fractured rocks was replaced by the injected water or that high-temperature water was replaced by the injected low-temperature water, indicating the existence of water flow. The diffraction stack migration revealed that Mqs1 and 2 were located at either end of a fracture zone (Fig. 4c). The water appeared to flow selectively via the fracture zone, as the increase in $V_{P}^{\text {inc. }}$ was restricted to the vicinity of the fracture zone.

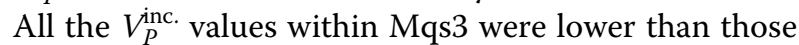
within Mqs1 and 2 (Fig. 4b). They were comparable to

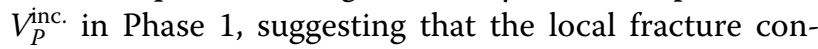
figuration around Mqs3 was similar to that during the pre-injection period. One possible scenario is that gas was a dominant phase around Mqs3 when the analyzed earthquakes occurred. Figure $4 \mathrm{~d}$ shows a cross section along the evolutional direction of Mqs3. Mqs3 appeared to evolve in a southwesterly direction (from Group 1 to Group 2; the temporal relationship is shown in Fig. 3b) within the local fracture, which is located at a depth of approximately $1-2 \mathrm{~km}$. The fracture could serve as the path for the fluid. Note, however, that $V_{P}^{\text {inc. indicated an }}$ absence of the liquid phase around Mqs3. It is difficult to explain Mqs3 using the water flow model.

\section{Other possible triggering processes}

Figure 3c shows the relationship between the intermittent events in Mqs2 and the pressure change rate per $6 \mathrm{~h}$ at the bottom of the Test Well (note that the pressure was not measured before the 15th day). The results show that the majority of microseismic events $(77 \%$, or 71 of 


\section{(b)}
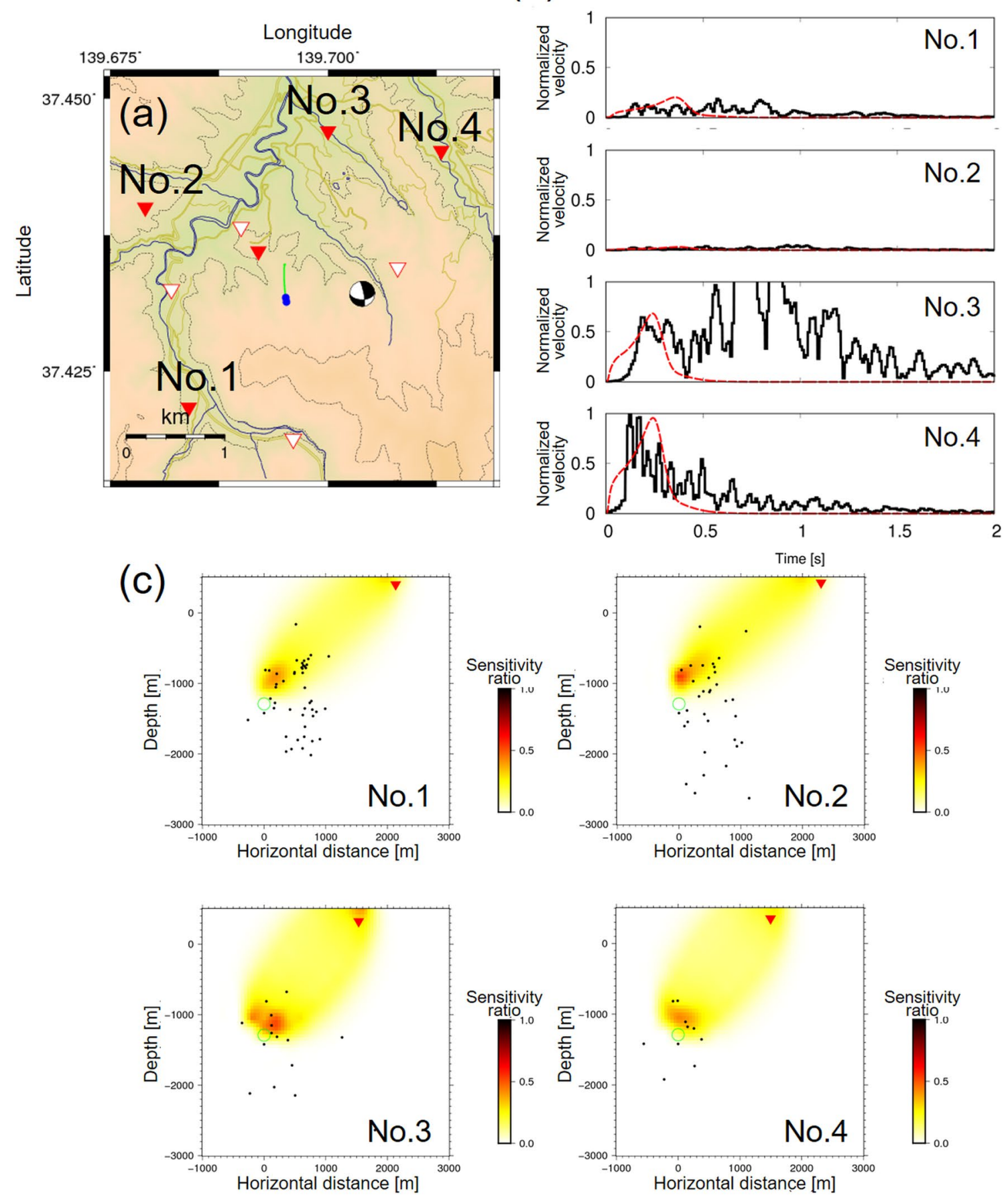

Fig. 2 Example of scattering analysis. a Location of the earthquake used for the example of scattering analysis; $\mathbf{b}$ observed (black line) and theoretical (red dashed line) waves; c sensitive area of initial $P$-waves for this example case projected on cross section including hypocenter (shown as circle) and receiver (shown as inverted triangle)

92 events) occurred within 7 days after the stress fluctuations resulting from the injection rate changes (the four 7-day intervals are shaded in gray in Fig. 3c). These 7-day intervals make up only $39 \%$ of the whole. Therefore, most microseismic events in Mqs2 appear to be triggered by stress fluctuation. However, the mechanism for 

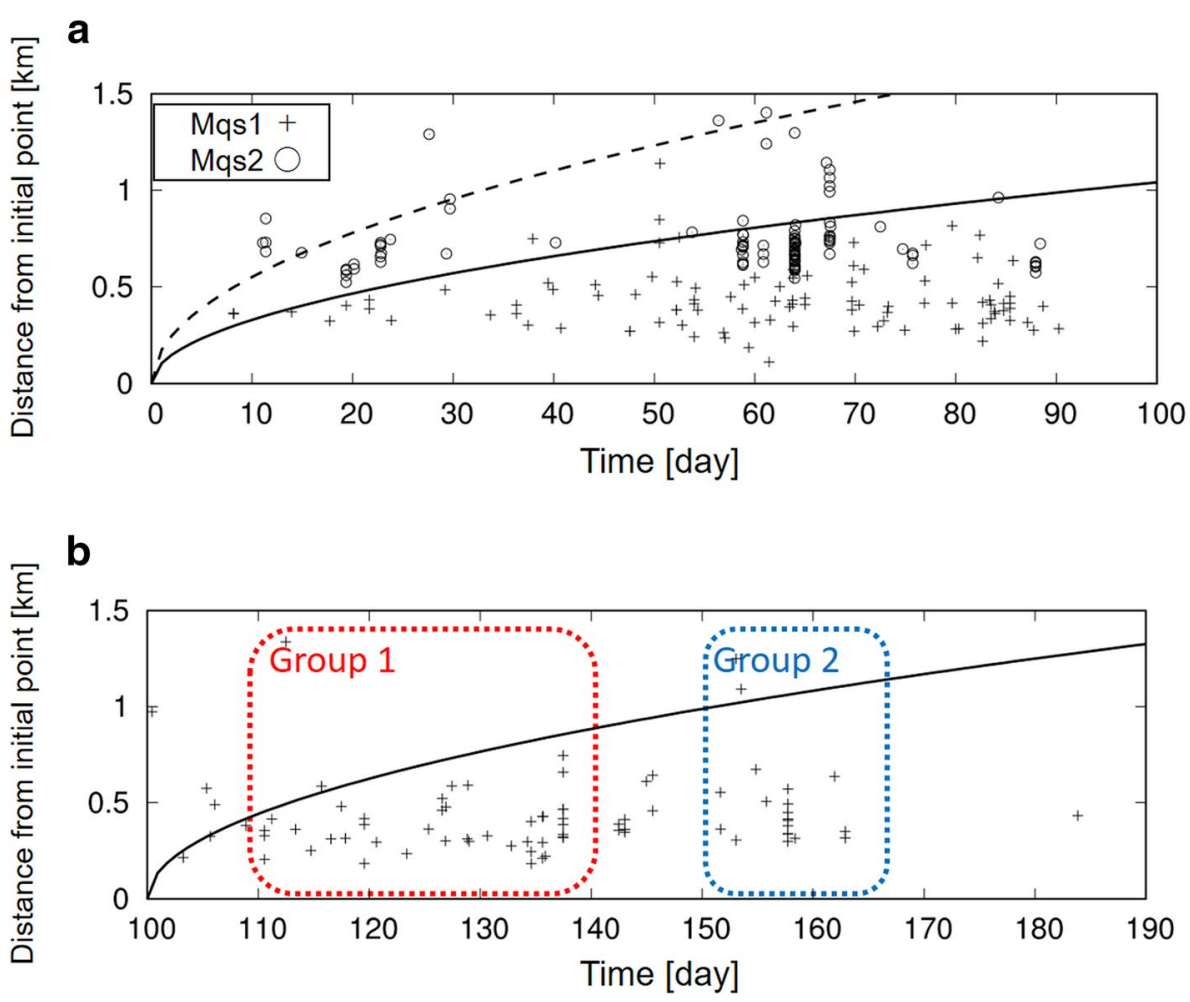

C

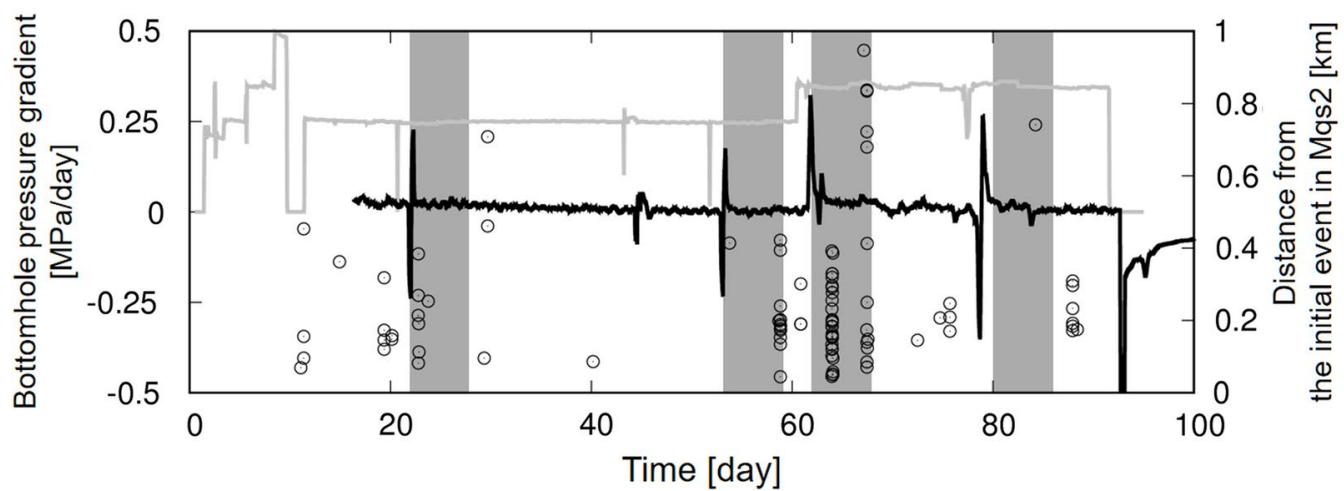

Fig. 3 Spatiotemporal distribution of microseismic events. a Results for Mqs1 and 2. The solid and dashed lines show diffusion curves for Mqs1 and $2 ; \mathbf{b}$ results for Mqs 3 with a diffusion curve. Groups 1 and 2 correspond to the groups of microseismic events shown in Fig. 4d. The horizontal axis shows days from the start of injection; the vertical axis shows distance from the initial point; c temporal relationship between Mqs 2 and pressure change rate at the bottom of the Test Well. The black line shows the pressure change rate (left vertical axis), and the circles show the spatiotemporal distribution of the microseismic events (right vertical axis). The length of the shaded areas is 7 days. Gray line represents injection rate in Fig. 1 a

the transfer of the stress fluctuation from the Test Well to Mqs2 is still unclear. Several studies (e.g., Häring et al. 2007; Asanuma et al. 2006; Baisch et al. 2010; Mukuhira et al. 2016) have pointed out that seismic events likely occur at the edge of reservoirs after shut-in owing to redistribution of the pore pressure at/after the injection is stopped. If the stress fluctuation due to the injection rate changes has an effect similar to that in the shut-in phase, it might be a trigger for the microseismic events that occurred at the periphery of the fracture zone (see Fig. 4c). If the diffusion process explains the transfer mechanism for the stress fluctuation, the hydraulic diffusivity $D$ in the fracture zone would be approximately $10^{-1}-10^{0} \mathrm{~m}^{2} / \mathrm{s}$ (the distance between the injection point and Mqs2 was roughly $1 \mathrm{~km}$; the time lag between the stress fluctuation and the microseismic events was 

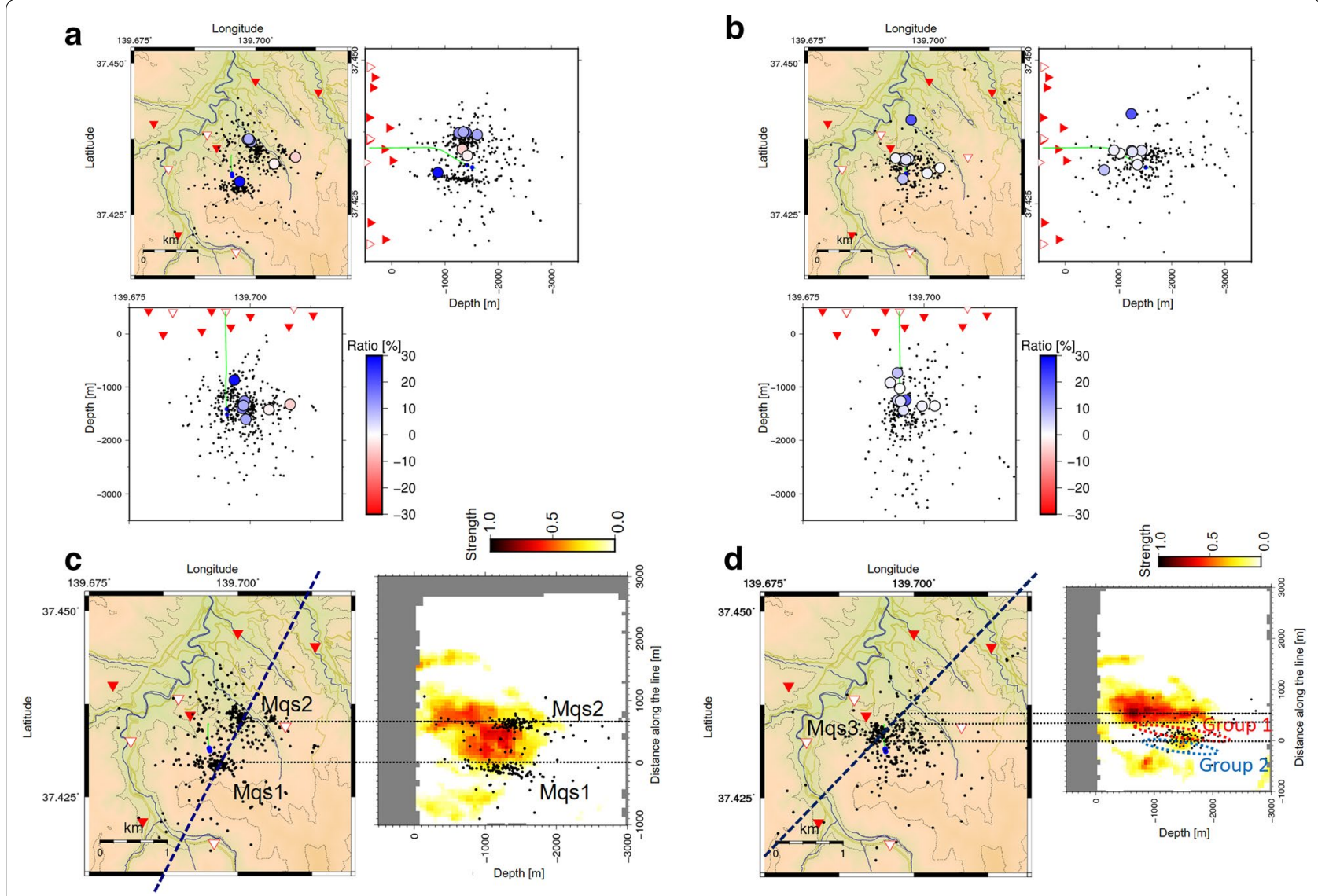

Fig. 4 Estimation of water flows around microseismic clusters. $\mathbf{a}, \mathbf{b}$ Variation in elastic wave velocity in rock fractures in $\mathbf{a}$ Phase 2 and $\mathbf{b}$ Phase 3 compared to the velocity in Phase 1. Velocity variation is shown by the color of each circle. Each circle is at the hypocenter of the analyzed earthquakes. $\mathbf{c}, \mathbf{d}$ Cross sections of the fractures and distributions of microseismic events along the blue dashed line in the horizontal map. Results for $\mathbf{c}$ Mqs 1 and 2 and $\mathbf{d}$ Mqs3. The color scales at the top of $\mathbf{c}$ and $\mathbf{d}$ indicate the probability that a fracture exists

several days). This diffusivity is much higher than the actual diffusivity around Mqs1, suggesting that the fracture zone is highly permeable. However, we should note that the stress fluctuation was $\sim 1 \mathrm{MPa}$ at most. It is thus not realistic to consider the diffusion process as the only trigger for Mqs2. Other factors caused by the injection rate changes may be additional triggers.

It was observed for Mqs3 that microseismic events in Group 1 were activated at the time of the phase transition of the fluid (from the liquid phase to the gas phase) at the bottom of the Test Well, which was confirmed by the temporal history of the temperature and pressure. Combining this fact with the $V_{P}^{\text {inc. }}$ results indicating that the fractured rocks within Mqs3 were perhaps dominated by water vapor, we surmise that the activation of Mqs3 was associated with phenomena induced by the phase transition (e.g., flashed steam from the injection point, a change in water volume). However, a causal relationship between the activation and the phase transition is unclear because the time of the phase transition at the location of Mqs3 is not available (the temporal history is available only for the bottom of the Test Well). Another problem is that the available observations (pressure and temperature) are not at the location of Mqs3 but at the bottom of the Test Well, and we currently do not have other evidence for the hypothesis.

\section{Summary and discussion}

Table 1 shows the possible triggering factors for Mqs1, 2, and 3. We found that Mqs1, which occurred in Phase 2 near the injection point, was consistent with a diffusion process. Scattering analysis revealed that the injected water appeared to flow through the fracture zone between Mqs1 and Msq2. Because the water flow did not induce microseismicity within the fracture zone, analysis of the hypocenter locations alone could not reveal the water flow. The scattering analysis was quite useful in the interpretation. One possible reason for the absence of seismicity is derived from the local stress orientation in the Okuaizu Geothermal Field. Most earthquakes in this 
Table 1 Summary of possible triggers for Mqs1, 2, and 3

\begin{tabular}{|c|c|c|c|}
\hline & Mqs1 & Mqs2 & Mqs3 \\
\hline Location & Periphery of fracture zone & Periphery of fracture zone & Inside fracture zone \\
\hline Period & During injection & During injection & After injection stopped \\
\hline Diffusion process & Yes & Yes & Yes \\
\hline Water flow model & Yes & Yes & Unclear \\
\hline Other possible triggering factors & & $\begin{array}{l}\text { Pressure fluctuation due to changes in injec- } \\
\text { tion rate }\end{array}$ & Phase transition of water (liquid to gas) \\
\hline
\end{tabular}

region have a strike angle along the NE-SW direction, although the strike angle of the fracture zone is along the SE-NW direction. Fractures in the zone might not slip easily.

Mqs2 may have been triggered by the water flow and the stress fluctuations resulting from changes in the injection rate. It seems that most of the microseismic events were triggered by the latter. We consider that a major mechanism by which the stress fluctuation propagated from the Test Well to Mqs2 is redistribution of the pore pressure, for example, in the shut-in phase. The saturated water in the fracture zone could be a mediator for the stress fluctuation. In Phase 3, Mqs2 was not activated, although the pressure at the Test Well was still changing. A possible reason for this inactivity is the absence of liquid water in the fracture zone. The water had likely been vaporized owing to a pressure drop and a temperature rise (as confirmed by data taken at the bottom of the Test Well) or had migrated farther away. Consequently, the stress fluctuation did not propagate. The fact that Mqs2 was reactivated during the second injection test, held in December 2015, supports our hypothesis. Note, however, that it took about 20 days before the vaporization was confirmed at the Test Well after the end of injection. The migration of the water may also take at least several days. Our hypothesis could not explain the low seismicity in Mqs2 for several days after the end of the injection. An important insight from our study is that Mqs2, which occurred on an existing fault during injection, could be controllable, as it may be caused by injection rate changes. For instance, avoiding sudden changes in the injection rate (e.g., a variation on the order of $10^{-1}$ MPa within several hours at the well bottom) may be a possible way to control the activity of Mqs2. However, it is necessary to investigate whether a fluctuation of $1 \mathrm{MPa}$ or less (the measured value at the well bottom) would trigger microseismicity several hundred meters away.

In Phase 3, a new microseismic cluster (Mqs3) was detected at a location different from those where Mqs1 and 2 occurred. The microseismic events could be explained by the diffusion process. In addition, Mqs3 was possibly triggered by a phase transition of water. To advance the discussion, the causal relationship between the microseismicity and phase transition needs to be investigated.

\section{Conclusions}

We applied a new method to estimate the temporal variations in the presence or absence of water in fractured rocks using scattered $P$-waves. The method is based on theoretical calculations by the Born approximation assuming a randomly inhomogeneous semi-infinite medium. By fitting theoretical waves to the observed waves, the elastic wave velocity in fractured rocks is calculated, and then, the presence of water is estimated. The method cannot treat multiple scattering because it is based on the Born approximation. Further, only uniform structures can be treated. However, these limitations may not significantly affect our results because we analyzed only the initial portion of the P codas. By combining the results with the high-resolution hypocenter determination yielded by the DD method, it is possible to estimate the presence of injected water around microseismic events. Our estimations provided a detailed image of the behavior of injected water and revealed that the triggering processes for microseismic events associated with water injection into geothermal reservoirs can differ depending on the location and time. We also showed the possibility of controlling the induced seismicity on existing faults. We believe that the knowledge gained from our study will lead to efficient steam production and optimal use of water injection in the Okuaizu Geothermal Field. Further application of our method to other geothermal fields will enable us to more fully understand the behavior of geothermal reservoirs associated with water injection.

\section{Authors' contributions}

$\mathrm{KO}$ played the main role in this study. $\mathrm{KO}$ analyzed and interpreted the observed microseismic events and drafted the manuscript. LY conducted the diffraction stack migration of seismic waves. HA participated in the design of the study and was involved in revising the manuscript. HA, TO, YA, and MT were involved in the design of the field surveys and made the data available. All authors read and approved the final manuscript. 


\begin{abstract}
Author details
${ }^{1}$ Renewable Energy Research Center, Fukushima Renewable Energy Institute National Institute of Advanced Industrial Science and Technology, 2-2-9 Machiikedai, Koriyama, Fukushima 963-0298, Japan. ${ }^{2}$ Geothermal Energy Research \& Development Co., Ltd., Shinkawa Nittei Annex Building, 22-4, Shinkawa 1-Chome, Chuo-ku, Tokyo 104-0033, Japan. ${ }^{3}$ Okuaizu Geothermal Co., Ltd, 1034-1 Sunakohara Kaminodaira, Yanaizu, Fukushima 969-7321, Japan. 4 Japan Oil, Gas and Metals National Corporation, Toranomon Twin Building 2-10-1 Toranomon, Minato-ku, Tokyo 105-0001, Japan.
\end{abstract}

\section{Acknowledgements}

We are grateful to the Geothermal Energy Research \& Development Co., Ltd. (GERD), and the Okuaizu Geothermal Co., Ltd. (OAG), for helping greatly with construction and maintenance of the microseismic monitoring system and making the dataset available. We thank Mr. Hiroki Hasegawa, Mr. Yujiro Mizusaki, and Mr. Eiji Kuwana for maintaining the microseismic monitoring system. We thank the anonymous reviewers for their many insightful suggestions. Several figures were prepared with Generic Mapping Tools (GMT) (Wessel et al. 2013).

\section{Competing interests}

The authors declare that they have no competing interests.

\section{Ethics approval and consent to participate}

Not applicable.

\section{Funding}

This work was partially supported by the "Technology to Evaluate and Manage Geothermal Reservoirs" project sponsored by Japan Oil, Gas and Metals National Corporation (JOGMEC).

\section{Publisher's Note}

Springer Nature remains neutral with regard to jurisdictional claims in published maps and institutional affiliations.

Received: 29 November 2017 Accepted: 23 January 2018

Published online: 01 February 2018

\section{References}

Asanuma H, Nozaki H, Uhara Y, Niitsuma H, Baria R, Wyborn D (2006) Spatial and temporal distribution of larger seismic events at European and Australian HDR sites. In: Proceedings of 31st workshop on geothermal reservoir engineering, Stanford, 30 January-1 February 2006

Asanuma H, Kumano Y, Niitsuma H, Schanz Y, Häring M (2008) Interpretation of reservoir structure from super-resolution mapping of microseismic multiplets from stimulation at Basel, Switzerland in 2006. Trans Geotherm Res Counc 32:53-58

Baisch S, Vörös R, Rothert E, Stang H, Jung R, Schellschmidt R (2010) A numerical model for fluid injection induced seismicity at Soultz-sous-Forêts. Int J Rock Mech Min Sci 47:405-413

Baria R, Baumgärtner J, Gérard A, Jung R, Garnish J (1999) European HDR research program at Soultz-sous-Forêts (France) 1987-1996. Geothermics 28:655-669

Evans KF, Genter A, Sausse J (2005) Permeability creation and damage due to massive fluid injections into granite at $3.5 \mathrm{~km}$ at Soultz: 1. Borehole observations J Geophys Res 110:B04203. https://doi. org/10.1029/2004JB003168

Fehler M, House L, Kaieda H (1987) Determining planes along which earthquakes occur: method and application to earthquakes accompanying hydraulic fracturing. J Geophys Res 92:9407-9414

Häring M, Ladner F, Schanz U, Spillman T (2007) Deep heat mining Basel, preliminary results. In: Proceedings of European geothermal conference, Unterhaching, 30 May-1 June 2007

Hollman KW, Rigby KW, O'Donnell M (1999) Coherence factor of speckle from a multi-row probe. In: Proceedings of IEEE ultrasonics symposium, Stateline, 17-20 Oct 1999
McClure M, Horne R (2011) Investigation of injection-induced seismicity using a coupled fluid flow and rate/state friction model. Geophysics 76(6):181-198

Mukuhira Y, Dinske C, Asanuma H, Ito T, Häring MO (2016) Pore pressure behavior at the shut-in phase and causality of large induced seismicity at Basel, Switzerland. J Geophys Res Solid Earth. https://doi. org/10.1002/2016JB013338

New Energy Development Organization (1997) Geothermal development promotion survey No. B-1 "Sarukuradake Area": geothermal development research report. http://geothermal.jogmec.go.jp/gathering/file/42.pdf. Accessed 29 Nov 2017

Okabe T, Kato M, Sato T, Abe Y, Asanuma H, Oishi T, Tosha T (2016) Current status of the EGS project for water injection in the superheated region at Okuaizu Geothermal Field in Japan. In: Proceedings of 38th New Zealand geothermal workshop, Auckland, 23-25 Nov 2016

Okamoto K, Tsuno S (2015) Investigation on relationship between epicentral distance and growth curve of initial P-wave propagating in local heterogeneous media for earthquake early warning system. Earth Planets Space. https://doi.org/10.1186/s40623-015-0339-3

Sato H (1984) Attenuation and envelope formation of three-component seismograms of small local earthquakes in randomly inhomogeneous lithosphere. J Geophys Res 89:1221-1241

Schaff DP, Bokelmann GHR, Ellsworth WL, Zanzerkia E, Waldhauser F, Beroza GC (2004) Optimizing correlation techniques for improved earthquake location. Bull Seismol Soc Am 94(2):705-721

Shapiro SA, Dinske C (2009) Fluid-induced seismicity: pressure diffusion and hydraulic fracturing. Geophys Prospect 57:301-310

Shapiro SA, Huenges E, Borm G (1997) Estimating the crust permeability from fluid-injection-induced seismic emission at the KTB site. Geophys J Int 131:F15-F18

Shapiro SA, Rothert E, Rath V, Rindschwentner J (2002) Characterization of fluid transport properties of reservoirs using induced microseismicity. Geophysics 67(1):212-220

Tromp J, Tape C, Liu Q (2005) Seismic tomography, adjoint methods, time reversal and banana-doughnut kernels. Geophys J Int 160:195-216

Waldhauser F, Ellsworth WL (2000) A double-difference earthquake location algorithm: method and application to the Northern Hayward Fault, California. Bull Seismol Soc Am 90(6):1353-1368

Wessel P, Smith WH, Scharroo R, Luis J, Wobbe F (2013) Generic mapping tools: improved version released. EOS Trans Am Geophys Union 94(45):409-410

Yi L, Okamoto K, Asanuma H (2017) Investigation on passive imaging methods of geothermal reservoirs by using the microearthquake events. In: Proceedings of 137th (2017 Fall) SEGJ conference, Yokohama, 8-10 Nov 2017

Yoshimoto K, Sato H, Ohtake M (1997) Three-component seismogram envelope synthesis in randomly inhomogeneous semi-infinite media based on the single scattering approximation. Phys Earth Planet Inter 104:37-61

\section{Submit your manuscript to a SpringerOpen ${ }^{\circ}$ journal and benefit from:}

- Convenient online submission

- Rigorous peer review

- Open access: articles freely available online

- High visibility within the field

- Retaining the copyright to your article

Submit your next manuscript at springeropen.com 